\title{
INSTRUMENTATION FOR THE MEASUREMENT OF HIGH ENERGY PHENOMENA ON NASA SPACECRAFT
}

\author{
ALBERT G. OPP and NANCY G. ROMAN \\ NASA, Washington, D.C., U.S.A.
}

High energy astrophysical observations supported by the National Aeronautics and Space Administration will be conducted primarily from the Small Astronomy Satellites (SAS) and the High Energy Astronomy Observatories (HEAO). At the present time, three Small Astronomy Satellites have been approved for flight. The first (SAS A) will carry a set of collimated proportional counters to conduct a high sensitivity, high spatial resolution, all sky survey for X-ray sources, to search for temporal variations in the source intensity, and to measure the spectral distribution of sources in the energy range 1 to $20 \mathrm{keV}$. The spacecraft will carry two collimator systems of 1 by $10 \mathrm{deg}$ and 10 by $10 \mathrm{deg}$ fullwidth. The proportional counters are gas-filled beryllium window tubes. SAS A is planned for launch in late 1970.

The objective of SAS B will be to search for celestial gamma-ray sources and regions and to measure the flux and energy spectrum of any sources located. A wire grid digitized spark chamber will be the prime detection instrument. The active area of the spark chamber is $25 \times 25 \mathrm{~cm}$. It is surrounded by an anti-coincidence dome to eliminate charged particle events. The spark chamber is triggered by a central plastic scintillator and Cerenkov counter. SAS B is planned for launch in 1971.

SAS C will carry four X-ray astronomy experiments. The instruments were selected to monitor the intensity and spectrum of galactic $X$-ray sources in the range 1 to $80 \mathrm{keV}$, to search for discrete X-ray emissions in the Magellanic Clouds, the Andromeda Nebula and in the galaxies of the Virgo Cluster, to monitor the fluctuations in SCO X-1 and to measure the galactic absorption of the diffuse X-ray background in the 1 to $10 \mathrm{keV}$ range. The instrumentation will consist of modulation collimators, slat collimators and proportional counters. SAS C is planned for launch in 1973.

The High Energy Astronomy Observatories (HEAO) are planned to accommodate relatively few, large, heavy experiments in X-ray and gamma-ray astronomy and high energy cosmic rays. HEAO A is visualized as an octagonal structure, approximately $9 \mathrm{~m}$ long $2.5 \mathrm{~m}$ across the flats. It will be launched by a Titan launch vehicle and will be able to accommodate up to $5500 \mathrm{~kg}$ of experiments. HEAO A is planned to survey the celestial sphere for X-ray and gamma-ray sources and high energy cosmic rays. The spacecraft will spin with a period of about $10 \mathrm{~min}$ around an axis approximately parallel to the Earth-Sun line. Subsequent HEAO spacecraft may have pointing up to one arc minute to explore in more detail specific sources of interest. Proposals for investigations on HEAO were received on 31 May 1970 and are presently under evaluation. The proposals that are selected will serve as the basis for design studies of the first four spacecraft. HEAO A is planned for launch in 1974, with subsequent missions approximately annually thereafter. 